\title{
Confecção de moldeira individual pela clonagem da prótese total provisória do paciente: relato de caso clínico
}

\author{
Confection of individual tray by duplicating of the provisional complete denture of the patient: clinical case \\ Confección de cubeta individual por la clonación de la prótesis total provisional del paciente: reporte de caso \\ Lorena Scaioni SILVA ${ }^{1}$ \\ Gustavo Porangaba MIRANDA ${ }^{1}$ \\ Aljomar José VECHIATO-FILHO ${ }^{2}$ \\ Fellippo Ramos VERRI ${ }^{3}$ \\ Victor Eduardo DE SOUZA BATISTA ${ }^{\mathbf{1}}$ \\ ${ }^{1}$ Faculdade de Odontologia de Presidente Prudente, Universidade do Oeste Paulista - UNOESTE, 19050.920 Presidente Prudente - SP, Brasil \\ ${ }^{2}$ Instituto do Câncer do Estado de São Paulo - ICESP, 01246-000, São Paulo -SP, Brasil \\ ${ }^{3}$ Faculdade de Odontologia de Araçatuba - FOA/UNESP, 16015-050, Araçatuba -SP, Brasil
}

\section{Resumo}

O objetivo do presente artigo foi relatar uma forma de confecção de prótese total utilizando a clonagem da prótese provisória com a finalidade de reduzir as etapas do tratamento. Paciente desdentada total superior e classe I de Kennedy inferior compareceu ao consultório apresentando inflamação severa do tecido periodontal, mobilidade grau III de todos os dentes e supuração à sondagem. Optou-se por realizar prótese total superior convencional e realização das exodontias dos dentes remanescentes, seguida de prótese total provisória e como tratamento definitivo mandibular uma prótese total convencional. Na primeira sessão foi realizada a reavaliação dos tecidos bucais, moldagem da prótese total superior para obter o modelo antagonista, seleção da cor do dente, registro para montagem no articulador semiajustável e a clonagem da prótese total inferior provisória. Após polimerizada, a réplica em resina acrílica incolor foi ajustada em boca e realizada a moldagem de borda com godiva em bastão, seguida da moldagem funcional com silicone de condensação. Ambos modelos foram montados no articulador para montagem dos dentes artificiais. A prova estética e funcional foi realizada na segunda consulta e na terceira a prótese foi instalada junto com as instruções de uso e higiene oral. Os controles foram realizados após 24 horas, 3,7 e 14 dias. O uso de uma técnica alternativa para confecção de uma moldeira individual pode reduzir o tempo clinico, trazendo assim vantagens tanto para o paciente como para o profissional, desde que haja um bom planejamento e conhecimento da técnica.

Descritores: Prótese Total; Técnica de Moldagem Odontológica; Materiais para Moldagem Odontológica.

\section{Abstract}

The aim of the present article was to report a way of making a complete denture by the duplication of the provisional complete denture in order to reduce the treatment steps. Patient with upper total edentulous and lower partial edentulous arches (Kennedy classe I) attended to dental office with severe inflammation of periodontal tissue, grade III mobility of all teeth and suppuration to probing. It was decided to perform conventional upper complete denture and to perform the extraction of the remaining teeth, followed by provisional complete denture and as a definitive mandibular treatment a conventional complete denture. The first session was performed re-evaluation of the oral tissues, dental impression of the upper complete denture to obtain the antagonist cast, color selection, registration for mounting the semi-adjustable articulator and duplicating of the provisional complete denture. After polymerization, the replica in colorless acrylic resin was adjusted in the mouth and the border molding with compound, followed by functional molding with condensation silicone. Both models were mounted on the articulator for artificial teeth arrangement. The aesthetic and functional test was performed in the second session and the complete denture was installed along with instructions for use and oral hygiene in the third session. Controls were performed after 24 hours, 3,7 and 14 days. The use of an alternative technique for fabrication of a custom tray may reduce the clinical time, as well as to offer benefits for both the patient and the professional; however, a good planning and knowledge of the applied technique is necessary.

Descriptors: Complete Denture; Dental Impression Technique; Dental Impression Materials.

\section{Resumen}

El objetivo del presente artículo fue relatar una forma de confección de prótesis total utilizando la clonación de la prótesis provisional con finalidad de reducir las etapas del tratamiento. Paciente desdentado total superior y clase I de Kennedy inferior asistió al consultorio presentando inflamación severa del tejido periodontal, movilidad grado III de todos los dientes y supuración al sondeo. Optó por realizar una prótesis total superior convencional y realización de exodontias de los dientes remanentes, seguida de prótesis total provisional y como tratamiento definitivo mandibular una prótesis total convencional. En la primera sesión se realizo reevaluación de los tejidos bucales, moldeo de la prótesis total superior para obtener el modelo antagonista, selección del color del diente, registro para montaje en articulador semiajustable y clonación de la prótesis total inferior provisional. Después de polimerizado, la réplica en resina acrílica incolora se ajustó en boca y realizó el molde de borde con godiva en bastón, seguida del moldeado funcional con silicona de condensación. Ambos modelos fueron montados en articulador para el montaje de los dientes artificiales. La prueba estética y funcional fue realizada en segunda consulta y en tercera la prótesis fue instalada junto con las instrucciones de uso e higiene oral. Los controles se realizaron después de 24 horas, 3,7 y 14 días. Una técnica alternativa para confección de una cubeta individual puede reducir el tiempo clínico, trayendo así ventajas tanto para el paciente y para profesional, siempre que haya una buena planificación y conocimiento de la técnica. Descriptores: Dentadura Completa; Técnica de Impresión Dental; Materiales de Impresión Dental.

\section{INTRODUÇÃO}

O edentulismo total é caracterizado pela perda de todos os elementos da arcada dentária, sendo considerado um dos maiores agravos à saúde bucal, devido a sua alta prevalência ${ }^{1,2}$. Segundo dados do Ministério da Saúde, pesquisa epidemiológica em saúde bucal no Brasil, realizada em 2010, detectou mais de 3 milhões de idosos $(15,8 \%)$ que necessitam de prótese total (PT) (em ambas arcadas dentárias) e outros 4 milhões $(21 \%)$ que precisam usar prótese parcial (em uma das arcadas) ${ }^{3}$. A ausência completa de dentes acarreta em danos físicos (reabsorção do rebordo), funcionais (diminuição da capacidade de mastigação e fonação), sociais e psicológico (reclusão social), ou seja, influencia na autoestima e qualidade de vida desses pacientes ${ }^{1,4,5}$. Para evitar e/ou minimizar esses danos, a reabilitação oral desses pacientes pode ser realizada através de prótese total convencional, sobredentadura implanto-suportada (overdenture sobre implante) sobre implantes ou próteses totais fixas sobre implante (também denominadas por próteses do tipo protocolo de Brånemark $)^{6-11}$, sendo a prótese total convencional a 
opção de tratamento mais utilizada para reabilitar pacientes edêntulos totais, pois proporciona resultados estéticos aceitáveis, devolve a função, tem um custo reduzido quando comparado à outra reabilitações, além de favorecer a higienização ao paciente $^{12-14}$.

Considerando a importância da reabilitação protética para a adequada função mastigatória e do sistema estomatognático, diferentes métodos de confecção de próteses foram desenvolvidos com a finalidade de contornar dificuldades encontrada por muitos pacientes ou diminuir o tempo clinico de sua confecção ${ }^{15,16}$, pois quando planejada e executada de maneira criteriosa, a maioria dos pacientes expressam satisfação com esse tipo de prótese ${ }^{17}$. As próteses totais são confeccionadas pela técnica "convencional" que exige uma ampla sequência de procedimentos clínicos e laboratoriais, sendo: moldagem de estudo, confecção de moldeira individual, moldagem funcional, individualização do arco facial, registo dos modelos em relação cêntrica e montagem dos dentes, prova estética e funcional, instalação e controles ${ }^{18-20}$.

Outra técnica que foi desenvolvida com o intuito de reduzir os custos e tempo de confecção, é a "simplificada", onde envolve um menor número de procedimentos clínicos ${ }^{21-24}$, tais como a confecção de bases de prova diretamente sobre modelos de estudos, eliminando uma segunda etapa de moldagem de maior precisão $0^{21,25}$; o não uso do $\operatorname{arco~facial~}^{22,26}$; realização da moldagem funcional e registro das relações maxilo-mandibulares em sessão única, utilizando a moldeira individual como base de prova $^{27}$ e, por fim, a duplicação da prótese do paciente, que se encontra em uso ${ }^{28-31}$.

A impressão fiel das áreas anatômicas através da moldagem é de suma importância, pois contribuem na estabilidade, retenção e conforto da prótese $^{32}$. Para a confecção de prótese total, dois tipos de moldagens geralmente são realizados: A moldagem anatômica, primária ou preliminar (estática), utilizando moldeira de estoque e tem como objetivo a reprodução da área chapeável de maneira geral, avaliação das inserções musculares e obtenção do modelo de estudo ${ }^{32,33}$; e a moldagem funcional, secundaria ou compressiva (dinâmica e seletiva), onde é realizada pressão em determinadas áreas e em outras o alívio ${ }^{34}$, com o objetivo de reproduzir os detalhes anatômicos com precisão, e para isso é utilizada uma moldeira individual, confeccionada previamente no modelo obtido pela moldagem anatômica ${ }^{32,35}$. As moldagens funcionais podem ser divididas em compressivas, não compressivas e com pressão seletiva ${ }^{36-39}$, e podem ser realizadas através da técnica de "boca aberta" ou "boca fechada"

Mediante a necessidade de extração dos elementos dentários remanescentes, a confecção de prótese total imediata é indicada para reabilitação temporária, na qual seguem os mesmos princípios e passos de uma prótese convencional, com exceção da prova estética e funcional, pois o aparelho é entregue na mesma sessão em que são realizadas as extrações ${ }^{35,41,42}$. Ainda neste contexto, as próteses provisórias possuem vantagens como: melhorar a estética do paciente, proteger as feridas cirúrgicas e promover uma melhor cicatrização, facilitar mastigação, evitar transtornos fonéticos, impedir o colapso labial e o afundamento das bochechas, bem como facilitar a determinação da dimensão vertical e manutenção da mesma, pois a dimensão natural do paciente pode ser aproveitada ou restabelecida, mantendo assim, a posição maxilo-mandibular adequada $^{32,35,43-45}$.

Diante do exposto, o objetivo do presente artigo foi relatar uma forma de confecção de prótese total utilizando a clonagem da prótese provisória do paciente com a finalidade de reduzir as etapas do tratamento.

\section{CASO CLÍNICO}

Paciente desdentada total superior e desdentada parcial inferior (classe I de Kennedy, dentes presentes de 33 a 43) compareceu ao consultório odontológico com queixa de dificuldade durante a alimentação. Durante a avaliação clínica observou-se inflamação severa do tecido periodontal, mobilidade grau III de todos os dentes, bem como supuração após a sondagem. Desta forma, as opções de tratamento para o arco maxilar foram: 1) realização de prótese total convencional; 2) realização de sobredentadura retida por implante ou 3) realização de prótese tipo protocolo. Para o arco mandibular, as seguintes opções de tratamento foram propostas: exodontia dos dentes $33,32,31,41,42$ e 43; seguida de instalação de prótese total imediata, sendo como opção definitiva: 1) a realização de prótese total convencional; 2) a realização de sobredentadura retida por implante ou 3) a realização de prótese tipo protocolo. Por fim, a paciente optou pela realização da prótese total superior convencional e realização das exodontias dos dentes 33 ao 43, seguida de prótese total provisória e como tratamento definitivo mandibular uma prótese total convencional.

Inicialmente, a prótese total superior definitiva foi confeccionada, de forma convencional, e instalada no mesmo dia das exodontias e instalação da prótese total inferior provisória. Nesta mesma consulta, a prótese total inferior provisória foi reembasada com material borrachóide provisório (Softconfort, Dencril, Pirassununga, São Paulo, Brasil) e após 24 h, 3 e 7 dias foram realizados os controles. Após 60 dias do último controle, a paciente retornou para a realização da prótese total inferior definitiva.

\section{- Etapas}

$\mathrm{Na}$ primeira consulta foi realizado o exame 
clínico para a reavaliação dos tecidos bucais (avaliação intraoral dos rebordos superiores e inferiores, bem como das próteses (Figuras 1 a 6) e moldagem da prótese total superior com alginato (Jeltrate, Dentsply sirona, York, Pensilvânia, EUA) com a finalidade de obtenção de modelo antagonista (Figuras 7,8 e 9). Além disso, foi realizada seleção da cor do dente. Nesta mesma consulta, registo para montagem em ASA superior foi realizado, bem como a clonagem da prótese total inferior provisória com a finalidade de obter uma réplica incolor em resina acrílica ativada quimicamente (RAAQ) para exercer a função de moldeira individual e facilitar o registro (Figuras 12 a 16). A polimerização da replica foi feita em panela elétrica polimerizadora analógica (Protécni, Protécni Equipamentos, Araraquara, São Paulo, Brasil), utilizando água fervendo por 5 minutos e resfriada em temperatura ambiente. Após polimerizada, o ajuste da réplica incolor em boca (moldeira individual) foi realizado, focando na estabilidade oclusal, tendo em vista que a dimensão vertical de oclusão e a posição em RC permanecessem mantidas. Além disso, desgastes nas bordas foram realizados para torná-las subestendidas (Figuras 17 e 18). Após criar o espaço nas bordas, a moldagem de borda com godiva em bastão (DFL, Nova Dfl Industria E Comercio SA, Curicica, Rio de Janeiro, Brasil) foi realizada (Figura 19). Em seguida, o adesivo universal para silicone (Universal adhesive, Heraeus Kulzer GmbH, Hanau, Hesse, Alemanha) foi aplicado na parte interna da replica e sobre a godiva $\mathrm{e}$, seguidamente, a realização da moldagem funcional propriamente dita com silicone de condensação (Xantopren LBlue, Heraeus Kulzer GmbH, Hanau, Hesse, Alemanha) utilizando a técnica da boca fechada (Figuras 20 e 21).

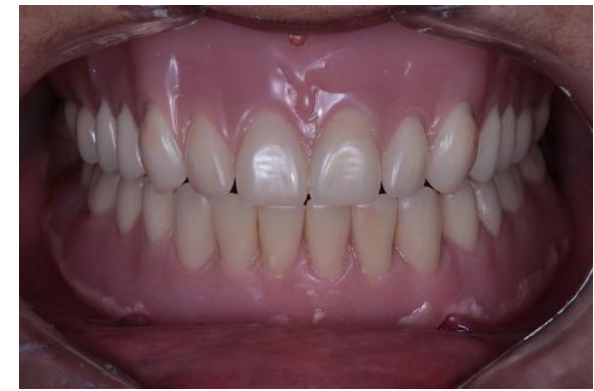

Figura 1: Exame clínico intraoral com a prótese total superior e a prótese total inferior provisória.

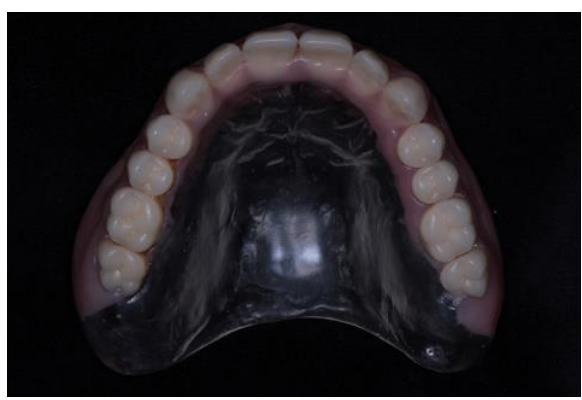

Figura 2: Vista oclusal da prótese total superior

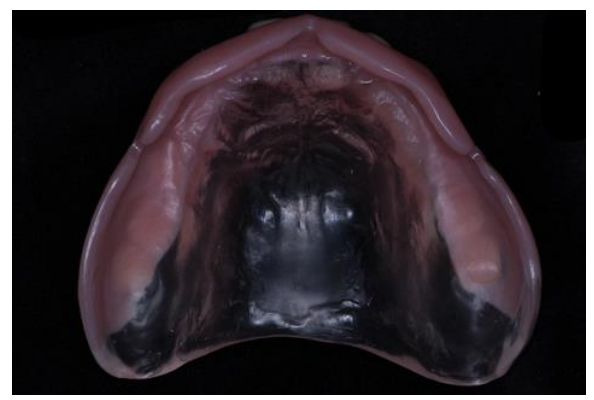

Figura 3: Vista interna da prótese total superior.

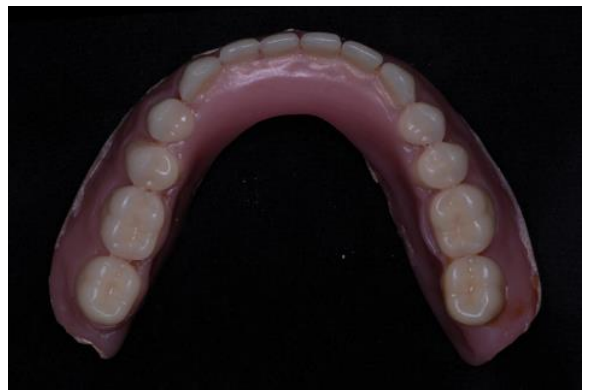

Figura 4: Vista oclusal da prótese total provisória.

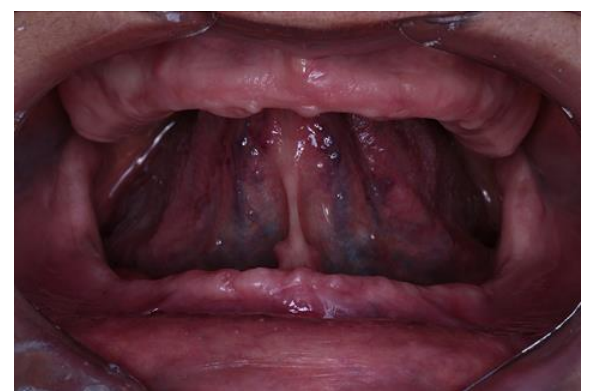

Figura 5: Rebordos superior e inferior sem as próteses.

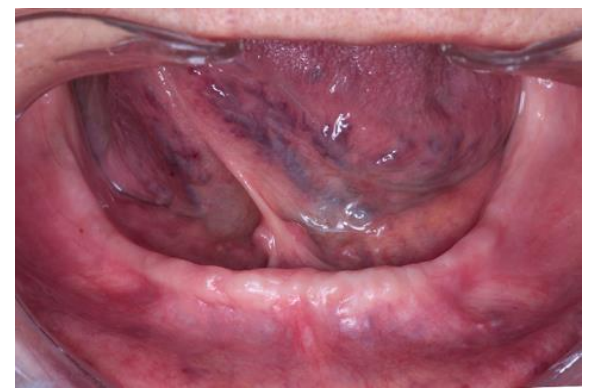

Figura 6: Rebordo inferior totalmente cicatrizado.

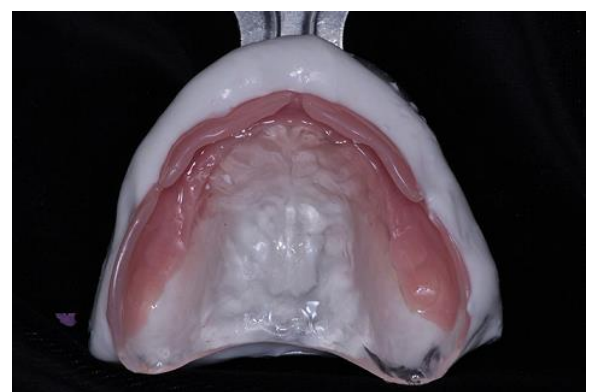

Figura 7: Moldagem com a prótese total em posição.

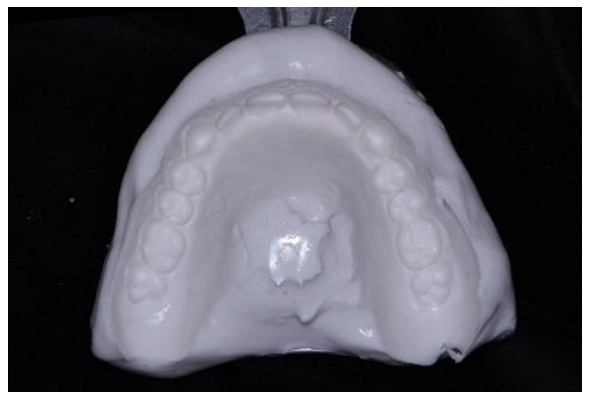

Figura 8: Molde da prótese total. 


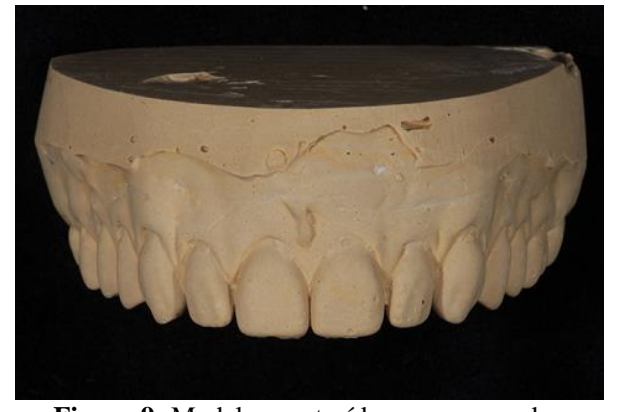

Figura 9: Modelo construído em gesso pedra.

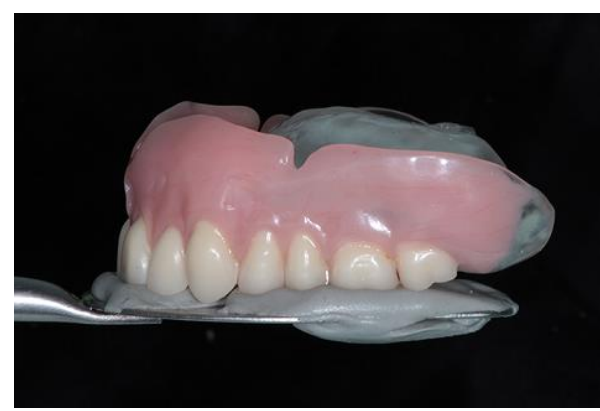

Figura 10: Registro para montagem em ASA: registro do arco superior utilizando a prótese total superior para marcar a oclusal.

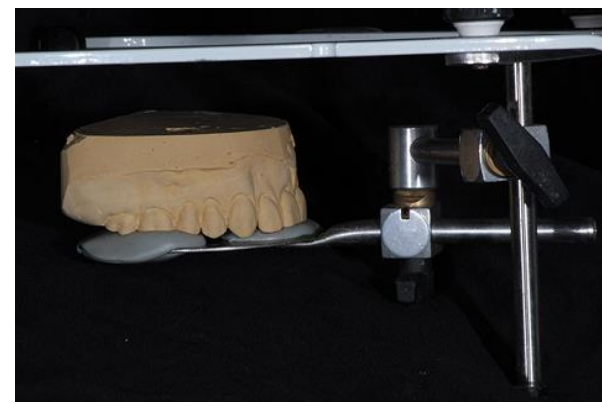

Figura 11: Adaptação do modelo antagonista ao registro para montagem em ASA.

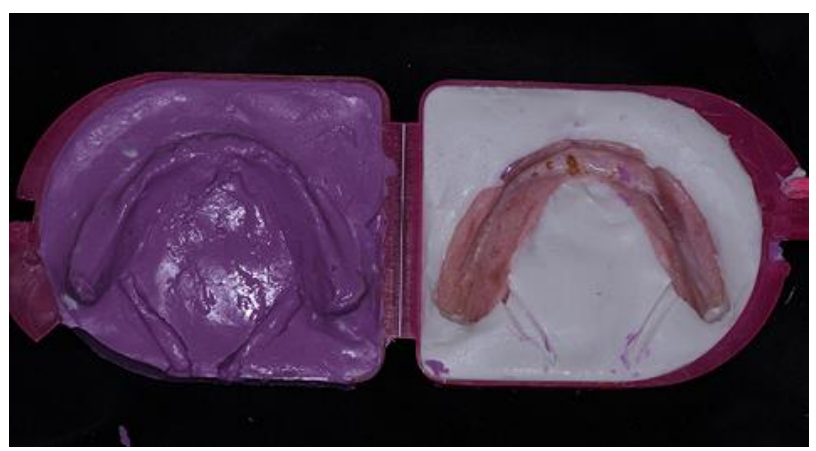

Figura 12: Clonagem da prótese total provisória inferior para confecção da réplica em RAAQ: prótese total inferior sendo copiada no dispositivo.

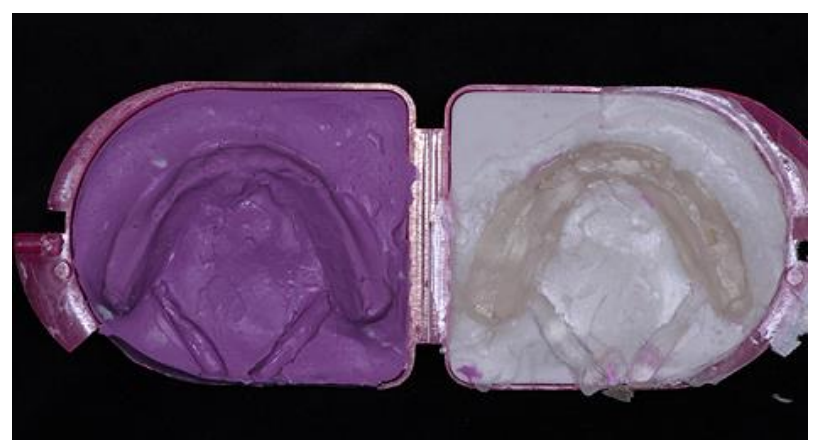

Figura 13: Clonagem da prótese total provisória inferior para confecção da réplica em RAAQ: espaço gerado após a remoção da prótese total inferior.

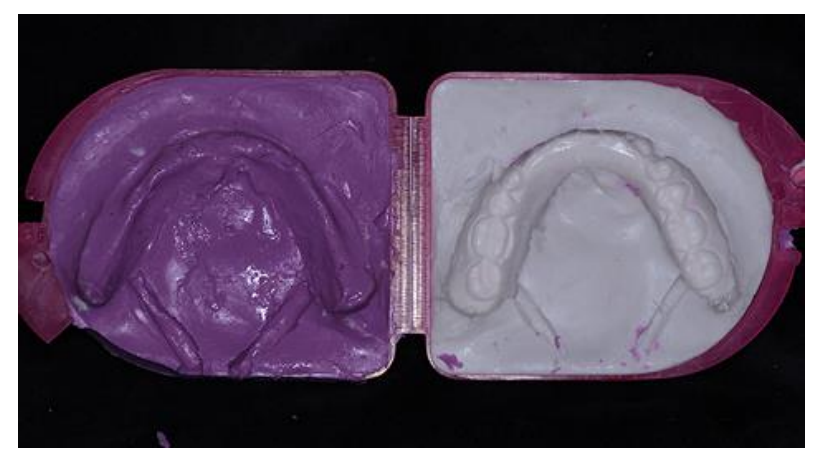

Figura 14: Clonagem da prótese total provisória inferior para confecção da réplica em RAAQ: espaço preenchido com RAAQ.

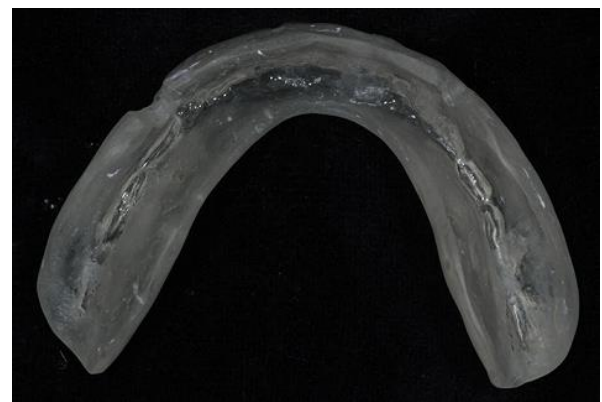

Figura 15: Clonagem da prótese total provisória inferior para confecção da réplica em RAAQ: réplica incolor finalizada vista interna.

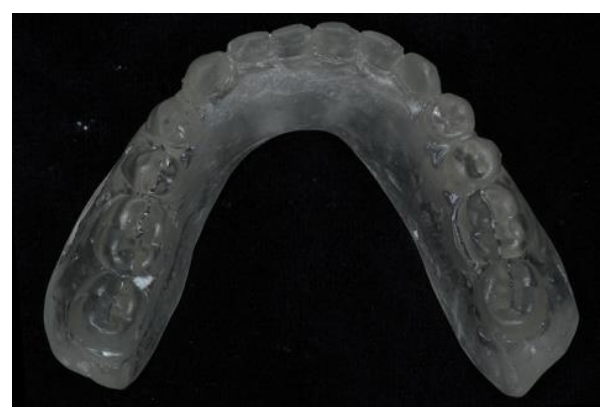

Figura 16: Clonagem da prótese total provisória inferior para confecção da réplica em RAAQ: réplica incolor finalizada vista oclusal.

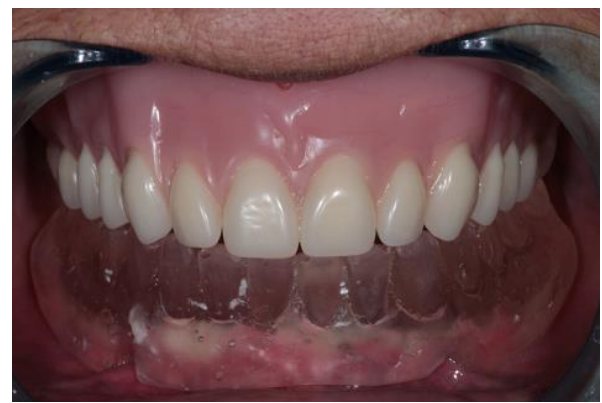

Figura 17: Réplica incolor em boca na qual não se observa espaço para o material de moldagem na borda.

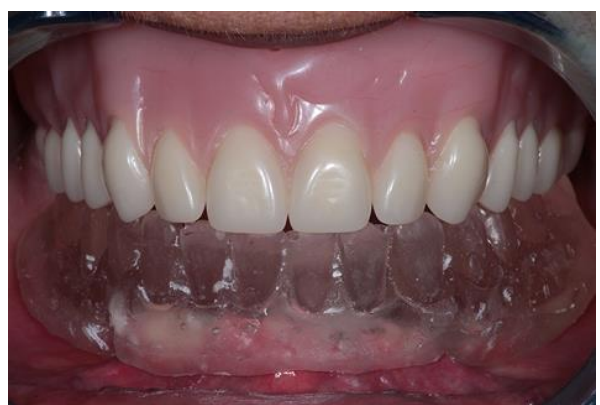

Figura 18: Réplica incolor em boca com bordas subestendidas. 


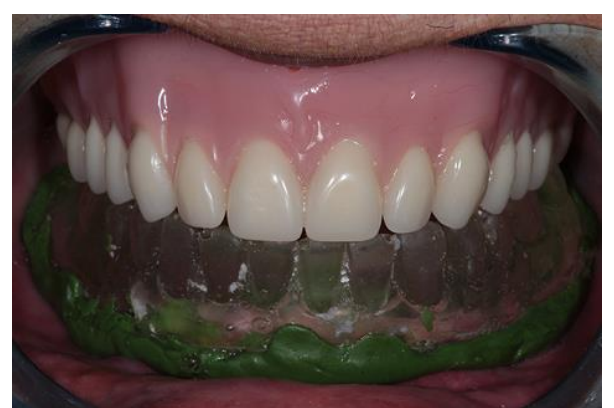

Figura 19: Moldagem de borda com godiva.

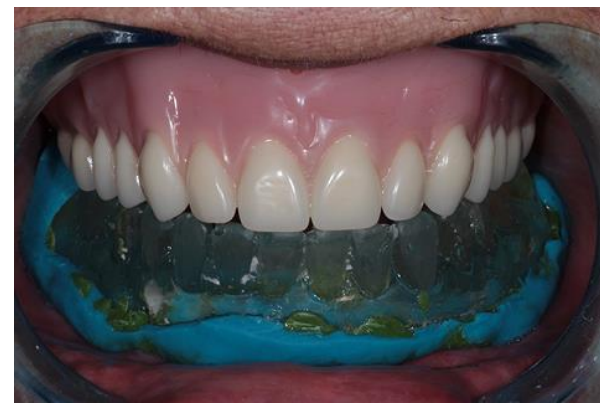

Figura 20: Moldagem funcional propriamente dita, boca fechada.

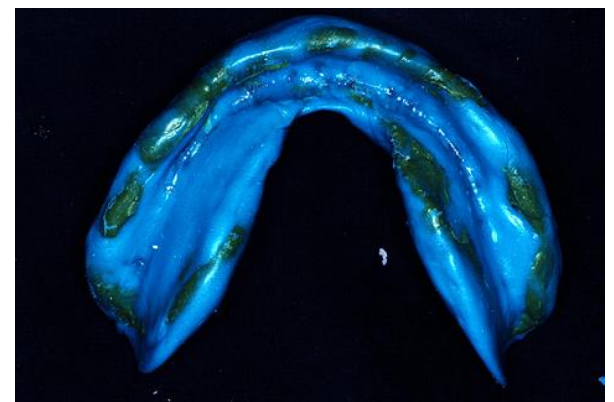

Figura 21: Vista interna do molde.

Os excessos de silicone foram recortados e o encaixotamento foi realizado para obtenção do modelo funcional em gesso pedra (Asfer, Asfer Indústria Química, São Caetano do Sul, São Paulo, Brasil) (Figura 22). O modelo inferior foi montado em articulador semi-ajustável, considerando o encaixe oclusal (Figuras 23 a 25).

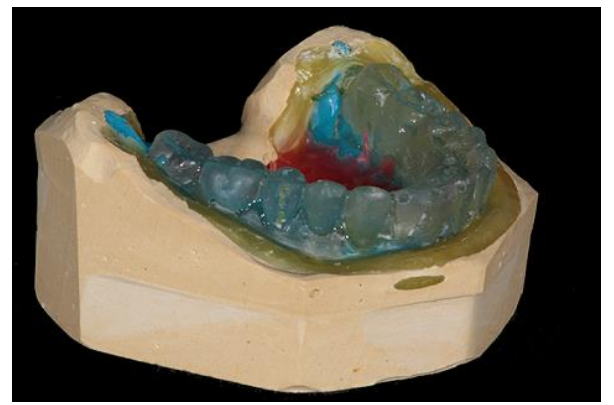

Figura 22: Obtenção do modelo de trabalho.

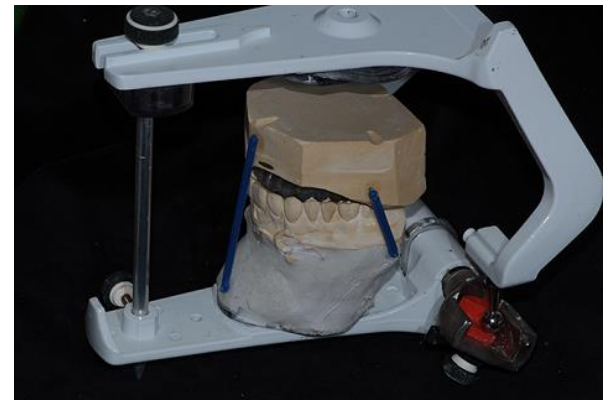

Figura 23: Modelo de trabalho com indexações sendo montado em ASA.

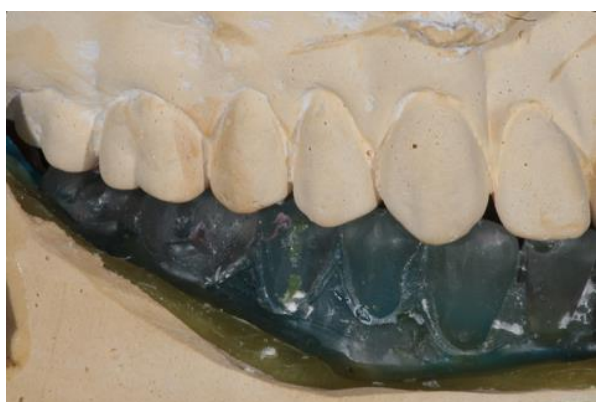

Figura 24: Montagem em ASA: encaixe preciso dos dentes.

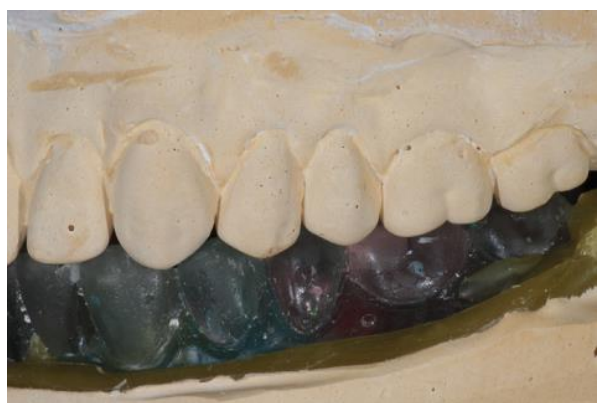

Figura 25: Montagem em ASA: encaixe preciso dos dentes.

Após montagem em ASA, a separação da moldeira individual da réplica foi realizada para análise do modelo funcional (Figuras 26 e 27). No laboratório, a base de prova foi confeccionada sobre esse modelo e posterior montagem dos dentes com esquema oclusal em oclusão bilateral balanceada (Figura 28).

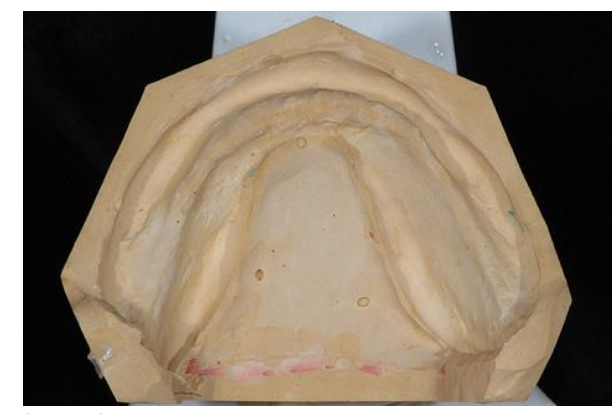

Figura 26: Modelo funcional após separação do molde.

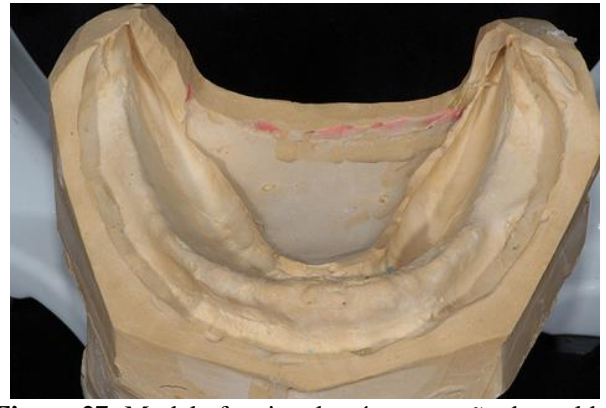

Figura 27: Modelo funcional após separação do molde.

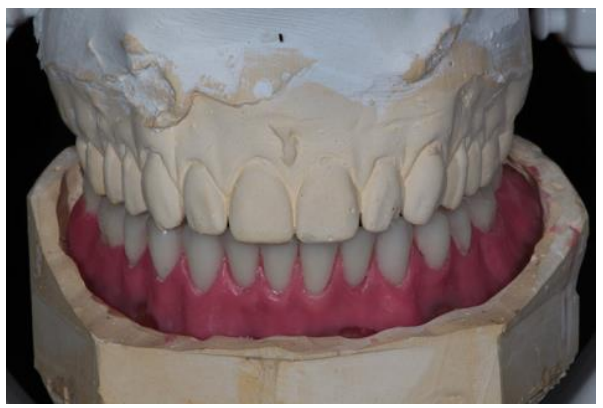

Figura 28: Montagem dos dentes em cera. 
A prova estética e funcional foi realizada na segunda consulta (Figura 29) e o paciente aprovou a cor, forma, bem como a montagem dos dentes, aprovando a finalização (acrilização) do trabalho protético. Desta forma, a cor da gengiva foi selecionada com base na prótese total superior e o trabalho foi enviado ao laboratório para a acrilização (Figuras 30 a 32). A prótese foi adaptada e instalada na terceira consulta (Figura 33), iniciando pela adaptação da prótese sobre a mucosa, identificando áreas de compressão em excesso com o auxilio de pasta zincoenólica (Lysanda, Lysanda Produtos Odontológicos, São Paulo, SP, Brasil) e eliminandoas com o auxílio de broca maxicut e ajuste oclusal. Por fim, um polimento foi realizado e instruções de uso e higiene foram reportados ao paciente. Os controles foram realizados após 24h, 3, 7 e 14 dias.

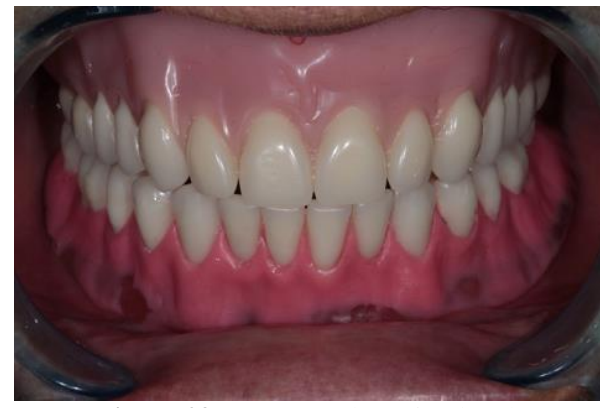

Figura 29: Prova estética e funcional.

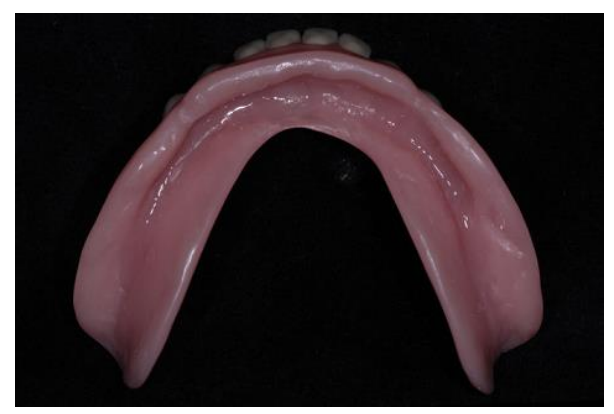

Figura 30: Prótese acrilizada.

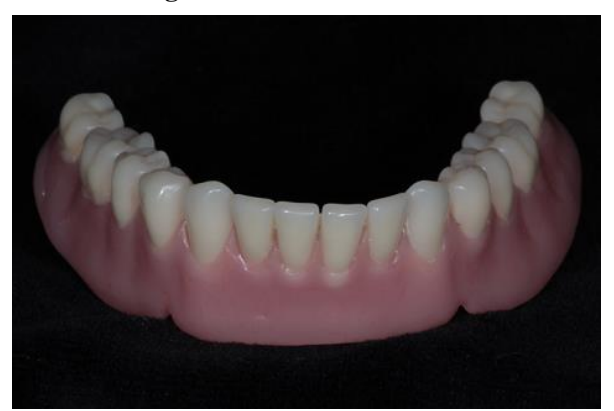

Figura 31: Prótese acrilizada.

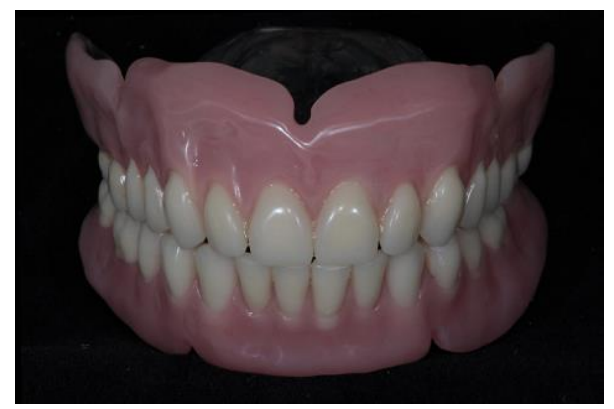

Figura 32: Prótese acrilizada.

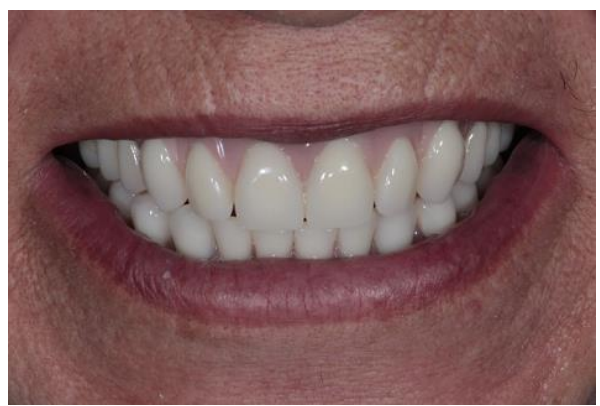

Figura 33: Instalação da prótese total inferior

\section{DISCUSSÃO}

Quando há necessidade de troca da prótese total antiga ou substituição da provisória por uma definitiva, a duplicação da prótese total que se encontra em uso pode ser indicada para ser utilizada como moldeira individual. A técnica proposta possui vantagens como: eliminação da fase de moldagem anatômica; facilitação da moldagem em oclusão por meio da técnica de boca fechada; são fáceis de manusear e possuem uma aparência agradável; no laboratório podem servir como referência para confecção da nova prótese, ou seja, requerem menor tempo de cadeira, tempo laboratorial e materiais ${ }^{28,46}$.

O uso do hidrocolóide irreversível para duplicação foi conveniente, pois é rápido, de fácil manipulação e baixo custo, no entanto, outros materiais de moldagem também podem ser utilizados nessa etapa, como o silicone de condensação ${ }^{32}$.

Para a moldagem funcional, o material de escolha foi o silicone de condensação fluído, pois é de fácil manuseio, tem sabor e odor agradável, custo moderado e é possível obter uma boa impressão do rebordo desdentado. Contudo, outros materiais também podem ser empregados, tais como o silicone de adição de consistência regular a leve, que apresenta excelente estabilidade dimensional e reprodução de detalhes, odor agradável e possibilita vários vazamentos, mas possui como desvantagem o alto custo; o poliéter, que é um material mais rígido, não libera subproduto na reação de presa, assim é possível obter excelentes modelos, contudo, também apresenta um custo elevado e menor resistência ao rasgamento; a pasta zincoenólica, que possui boa estabilidade dimensional, bom escoamento e fluidez e um ótimo custo/benefício, não obstante, desvantagens como ausência de flexibilidade, tempo de presa e trabalho reduzido e causa desconforto por ser muito pegajoso são apresentadas e, por fim, a mercaptana, por ser um material de fácil utilização, apresentar boa resistência ao rasgamento e boa reprodução dos detalhes, porém apresenta um odor desagradável bem como uma memória elástica deficiente ${ }^{47,48}$.

A técnica apresenta algumas limitações, sendo necessário a avalição de alguns critérios como a preexistência de uma prótese antagonista adequada; a prótese que será clonada deve apresentar a dimensão vertical e relação central adequadas; o 
profissional deve ter o domínio da técnica e conhecimento dos materiais utilizados. Além disso, é necessário destacar a problemática de confeccionar a réplica em RAAQ e realizar a moldagem na mesma consulta. Sabe-se que a RAAQ sofre alteração dimensional durante as 24 horas após sua polimerização, gerando a distorção no seu molde e, consequentemente, no seu modelo de trabalho ${ }^{49}$.

Como alternativa para evitar essa contração, a réplica de RAAQ deve ser colocada em água fervente por 5 minutos e depois resfriada à temperatura ambiente ${ }^{50}$. Além disso, vale ressaltar que ainda é possível a utilização de aparelhos protéticos provisórios que apresentam alguns problemas que requerem correção para a obtenção de próteses funcionais ideias como a correção da articulação com os dentes antagonistas e curvatura do arco, por exemplo. Nessas situações, o profissional deverá realizar o registro oclusal após moldagem e solicitar a montagem dos dentes em plano de cera para a realização da prova estética-funcional das próteses. Mesmo diante desse cenário, a técnica discutida no presente artigo elimina a moldagem preliminar e necessidade de confecção de moldeira individual.

\section{CONSIDERAÇÕES FINAIS}

O uso de uma técnica alternativa para confecção de uma moldeira individual pode reduzir o tempo clinico, trazendo assim vantagens tanto para o paciente como para o profissional, desde que haja um bom planejamento e conhecimento da técnica.

\section{REFERÊNCIAS}

1. Emami E, Souza RF, Kabawat M, Feine JS. The impact of edentulism on oral and general health. Int J Dent. 2013;2013:498305

2. Williams SE, Slice DE. Influence of edentulism on human orbit and zygomatic arch shape. Clin anat. 2014;27(3):408-16.

3. Brasil. Ministério da Saúde. Projeto SB Brasil 2010: Brasil Sorridente - A saúde bucal levada a sério. Brasília: Ministério da Saúde; 2010.

4. Petersen PE. The World Oral Health Report 2003: continuous improvement of oral health in the 21st century - the approach of the WHO Global Oral Health Programme. Community Dent Oral Epidemiol. 2003;31(Suppl 1):3-23.

5. Agostinho ACMG, Campos ML, Silveira JLGC. Edentulismo, uso de prótese e autopercepção de saúde bucal entre idosos. Rev odontol UNESP. 2015; 44(2):74-9.

6. Di Fiori SR, Di Fiori M, Di Fiori AP. Atlas de prótese parcial removível. São Paulo: Santos; 2010.

7. Telles D. Prótese total: convencional e sobre implantes. São Paulo: Santos; 2011.

8. Carlsson GE, Omar R. The future of complete dentures in oral rehabilitation. A critical review. $\mathbf{J}$ Oral Rehabil. 2010;37(2):143-56.
9. Mericske-Stern RD, Taylor TD, Belser U. Management of the edentulous patient. Clin Oral Implants Res. 2000;11(1):108-25.

10. Telles D, Coelho AB. Próteses sobre implantes. Rio de Janeiro: SobreImplantes.com; 2006.

11. Calvani L, Michalakis K, Hirayama H. The influence of full-arch implant-retained fixed dental prostheses on upper lip support and lower facial esthetics: preliminary clinical observations. Eur J Esthet Dent. 2007;2(4):420-28.

12. Cooper LF. The current and future treatment of edentulism. J Prosthodont. 2009;18(2):116-22

13. Thomason JM, Lund JP, Chehade A, Feine JS. Patient satisfaction with mandibular implant overdenture and conventional denture 6 months after delivery. Int J Prosthodont. 2003;16(5):467-73.

14. Silva-Junior MF, Fonseca EP, Batista MJ, Sousa MLR. Spatial distribuition of tooth loss in a population of adults. RGO. 2017;65(2):115-20.

15. Ettinger RL. Managing and treating the atrophic mandible. J Am Dent Assoc. 1993;124(7):234-41

16. Lindquist TJ, Narhi TO, Ettinger RL. Denture duplication technique with alternative materials. J Prosthet Dent. 1997;77(1):97-8.

17. Barros AWP, Porto E, Lima JFS, Brito NMSO, Soares RSC. Steps for biomodel acquisition through addtive manufacturing for health. RGO. 2016;64(4):442-6.

18. Watamabe Y. Observation of horizontal mandibular positions in an edentulous patient using a digital gothic arch tracer: a clinical report. J Prosthet Dent. 2004;91(1):15-9.

19. Williamson RA, Williamson AE, Bowley J, Toothaker R. Maximizing mandibular prosthesis stability utilizing linear occlusion, occlusal plane selection, and centric recording. J Prosthodont. 2004;13(1):55-61.

20. Paixão F, Silva WAB, Silva FA, Ramos GG, Cruz MVJ. Evaluation of the reproducibility of two techniques used to determine and record centric relation in Angle's class I patients. J Appl Oral Sci. 2007;15(4):275-79.

21. Duncan JP, Taylor TD. Simplified complete dentures. Dent Clin North Am. 2004; 48(3):25-40.

22. Nascimento DFF, Patto RBL, Marchini L, Cunha VPP. Double-nlind study for evaluation of complete dentures made by two techniques with and without face-bow. Braz J Oral Sci. 2004;3(9):439-45.

23. Kawai Y, Murakami H, Takanashi Y, Lund JP, Feine JS. Efficient resource use in simplified complete denture fabrication. J Prosthodont. 2010;19(7):512-16.

24. Heydecke G, Vogeler M, Wolkewitz M, Türp JC, Strub JR. Simplified versus comprehensive fabrication of complete dentures: patient ratings of denture satisfaction from a randomized crossover trial. Quintessence Int. 2008;39(2):107-16. 
25. Duncan JP, Taylor TD. Teaching an abbreviated impression technique for complete dentures in an undergraduate dental curriculum. J Prosthet Dent. 2001;85(2):121-25.

26. Heydecke G, Akkad AS, Wolkewitz M, Vogeler M, Türp JC, Strub JR. Patient ratings of chewing ability from a randomised crossover trial: lingualised vs. first premolar/canine-guided occlusion for complete dentures. Gerodontology. 2007;24(2):77-86.

27. Ansari IH. A one-appointment impression and centric relation record technique for compromised complete denture patients. J Prosthet Dent. 1997;78(3):320-23.

28. Davis DM, Watson RM. A retrospective study comparing duplication and conventionally made complete dentures for a group of elderly people. Br Dent J. 1993;175(2):57-60.

29. Clark RfK. The future of teaching of complete denture construction to undergraduates. Br Dent J. 2002;193(1):13-4.

30. Ellis JS, Pelekis ND, Thomason JM. Conventional rehabilitation of edentulous patients: the impact on oral health-related quality of life and patient satisfaction. J Prosthodont. 2007;16(1):37-42.

31. Rodrigues AHC, Morgano SM. An expedited technique for remaking a single complete denture for an edentulous patient. J Prosthet Dent. 2007;98(3):232-34.

32. Tamaki T. Dentaduras completas, 4. ed. São Paulo: Sarvier; 1983.

33. Reis JMSN, Perez LECP, Nogueira S, Ariolli Filho JN, Moll Júnior FA. Moldagem em prótese total: uma revisão de literatura. RFO. 2007;12(1):70-4.

34. Bonachela WC, Rosseti PHO. Overdentures: das raízes implantadas osseointegrados planejamentos, tendências e inovações. São Paulo: Santos; 2002.

35. Telles D. Prótese total - convencional e sobre implantes. 2. ed. São Paulo: Santos; 2010.

36. Felton DA, Cooper LF, Scurria MS. Predictable impression procedures for complete dentures. Dent Clin North Am. 1996; 40(1):39-51.

37. Lee RE. Mucostatics. Dent Clin North Am. 1980; 24(1):81-96.

38. DeFranco RL, Sallustio A. An impression procedure for the severely atrophied mandibule. J Prosthet Dent. 1995;73(6):574-77.

39. Tripathi A, Singh SV, Aggarwal H, Gupta A. Effect of mucostatic and selective pressure impression techniques on residual ridge resorption in individuals with different bone mineral densities: A prospective clinical pilot study. J Prosthet Dent. 2019;121(1):90-4.

40. Levin BL. Impressions for complete dentures. Chicago: Quintessence; 1984.
41. Turano JC, Turano LM. Consertos e Reembasamentos. Fundamentos da prótese total, 7.ed. São Paulo: Santos; 2004.

42. Caputi S, Murmura G, Ricci L, Varvara G, Sinjari B. Immediate denture fabrication: a clinical report. Ann Stomatol (Roma). 2013;4(3-4):273-77.

43. Seals Jr RR, Kuebker WA, Stewart KL. Immediate complete dentures. Dent Clin North Am. 1996; 40(1):151-67.

44. Cerveira Neto H. Prótese total imediata. 2. ed. São Paulo: Pancast Editorial; 1987.

45. Marchini L, Santos JFF, Cunha VPP. Prótese total imediata: considerações clínicas. Robrac. 2000;9(27):45-9.

46. Wagner AG. Making duplicate dentures for use as final impression trays. J Prosthet Dent. 1970;24(1):111-13.

47. Anusavice KJ. Phillips: materiais dentários. 10.ed. Rio de Janeiro: Guanabara Koogan;1998.

48. Elie E. Daou. The elastomers for complete denture impression: a review of the literature. Saudi Dent J. 2010;22(4):153-60.

49. Goldfogel M, Harvey WL, Winter D.Dimensional change of acrylic resin tray materials. J Prosthet Dent. 1985;54(2):284-86.

50. Pagniano RP, Scheid RC, Clowson RL, Dagefoerde RO, Zardiackas LD. Linear dimensional change of acrylic resins used in the fabrication of custom trays. J Prosthet Dent. 1982;47(3):279-83.

\section{CONFLITO DE INTERESSES}

Os autores declaram não haver conflitos de interesse.

\section{AUTOR PARA CORRESPONDENCIA}

Victor Eduardo de Souza Batista

victor_edsb@hotmail.com

Submetido em 24/01/2019

Aceito em 12/03/2019 